\title{
The optimization of in vitro high-throughput chemical lysis of Escherichia coli. Application to ACP domain of the polyketide synthase ppsC from Mycobacterium tuberculosis
}

\author{
Pawel Listwan · Jean-Denis Pédelacq · Meghan Lockard • Carolyn Bell • \\ Thomas C. Terwilliger · Geoffrey S. Waldo
}

Received: 3 September 2009/ Accepted: 30 December 2009/Published online: 13 January 2010

(C) The Author(s) 2010. This article is published with open access at Springerlink.com

\begin{abstract}
Protein production in Escherichia coli involves high-level expression in a culture, followed by harvesting of the cells and finally their disruption, or lysis, to release the expressed proteins. We compare three high-throughput chemical lysis methods to sonication, using a robotic platform and methodologies developed in our laboratory [1]. Under the same expression conditions, all lysis methods varied in the degree of released soluble proteins. With a set of 96 test proteins, we used our split GFP to quantify the soluble and insoluble protein fractions after lysis. Both the amount of soluble protein and the percentage recovered in the soluble fraction using SoluLyse ${ }^{\circledR}$ were well correlated with sonication. Two other methods, Bugbuster $\AA$ and lysozyme, did not correlate well with sonication. Considering the effects of lysis methods on protein solubility is especially important when accurate protein solubility measurements are needed, for example, when testing adjuvants, growth media, temperature, or when establishing the effects of truncation or sequence variation on protein stability.
\end{abstract}

Keywords Automation · High-throughput screening · Split GFP · Cell lysis · Protein solubility · Robotics

P. Listwan $(\bowtie) \cdot$ J.-D. Pédelacq · M. Lockard · C. Bell ·

T. C. Terwilliger - G. S. Waldo

Bioscience Division, MS-M888, Los Alamos National

Laboratory, Bikini Atoll Rd, SM30, Los Alamos,

NM 87545, USA

e-mail: plistwan@lanl.gov

J.-D. Pédelacq

Institut de Pharmacologie et de Biologie Structurale (IPBS),

CNRS, Toulouse, France

J.-D. Pédelacq

UPS, IPBS, Université de Toulouse, Toulouse, France

\begin{abstract}
Abbreviations
GFP Green fluorescent protein

SAMI SAGIAN ${ }^{\mathrm{TM}}$ automated method interface

HTP High-throughput
\end{abstract}

\section{Introduction}

One of the benefits afforded by the International Structural Genomics Organization initiative (ISGO) is the development of new automated, high-throughput (HTP) protein production technologies. Soluble protein production for structural and functional determination is essential, yet is also one of the most difficult parts of the sequence-tostructure pipeline. The Protein Structure Initiative (PSI) in United States, the Structural Proteomics in Europe (SPINE), and other partner programs around the world have established a variety of automated methodologies for processing a large number of protein constructs [2-8]. Despite years of research and development in protein production, automation, and HTP technology, no single cell disruption methodology exists that satisfies the needs of all structural genomics laboratories.

Automated, HTP protein production as the instrument for structure determination is a complex, multistep process that requires the optimization of each individual task. Escherichia coli continues to be a popular host for protein expression despite the large proportion of the recombinant proteins that often accumulate as aggregates or inclusion bodies [9]. Changing the expression host to insect cells, baculovirus, cell-free expression or mammalian expression often presents additional problems. For this reason many laboratories and commercial institutions direct great efforts 
to improve bacterial expression strains, vector systems, and other factors that improve recombinant protein expression and solubility.

One of the most crucial steps to be optimized in the protein production process is bacterial cell lysis. Although bacterial cell lysis does not influence protein expression, it can have an effect on protein solubility by affecting the physicochemical properties of the protein. Conventional biochemistry laboratories working with a few protein targets can test and optimize many lysis methods. These include techniques such as mechanical cell disruptione.g. sonication, french press, and freeze-thaw, and chemical lysis using different buffer composition, lysozyme, or commercially available detergent reagents. Cell lysis can also include a combination of the mechanical and chemical lysis, e.g. lysozyme with freeze-thaw cycles. The preferred method, or "gold standard", for bacterial lysis on the small or standard laboratory scale production is sonication. It relies on the mechanical disruption of the bacterial cell wall. The expressed protein is not affected by any solubilizing lysis agents, like detergents, that can affect solubility or stability [10, 11]. On the other hand, when hundreds or thousands of different proteins, truncation, or sequence variants are screened, only a few lysis methods can be reasonably employed. Sonication becomes more problematic when hundreds of proteins need to be released from the bacteria using automated, HTP liquid handling platforms. Although there are HTP sonicators available on the market, e.g. SonicMan (MatriCal, Spokane, WA), most structural genomics liquid handling platforms were established before the availability of the HTP sonicators. Additionally, the selection of high-throughput sonicators is still very limited and costly [consequently are often difficult to integrate with current laboratory setups]. For this reason many HTP laboratories choose to optimize lysis conditions by chemical means.

As a member of the Integrated Center for Structure and Function Innovation (ISFI), part of the PSI Specialized Center Program, we are focused on developing methods that overcome bottlenecks in soluble protein production and protein crystallization. The split-GFP technology developed in this laboratory [12-16] has recently been used to develop an automated, HTP solubility screening assay, allowing us to process and screen thousands of protein constructs for solubility in a few days [1]. Briefly, split GFP technology uses highly engineered, self-complementing GFP fragments originally derived from "superfolder" GFP: a 15 amino acid GFP "tagging" fragment-strand 11 (S11 or GFP 11) and a GFP 1-10 "detector" fragment. The GFP S11 fragment is fused to the C-terminus of the protein of interest in a pTET plasmid. GFP 1-10 is separately expressed in a pET plasmid. The S11 fragment is available for complementation by the GFP 1-10 fragment only if the protein of interest is stable and soluble. This spontaneous complementation leads to formation of the fluorescent GFP betabarrel.

Screening terminal deletion libraries with the split GFP in order to identify compact, soluble domains can facilitate structural study of large, multidomain proteins. The measured solubility and sequenced ends of each fragment from the library are mapped onto the protein's sequence, providing a comprehensive roadmap of soluble expression as a function of $5^{\prime}$ and $3^{\prime}$ construct ends.

The objective of library screening is to evaluate the intrinsic solubility and stability of each member. Even single amino acid extensions or deletions at either end of the protein can profoundly affect expression. It is important to control the effects of chemical lysis on protein stability in order to reliably and accurately measure the effects of amino acid mutations or terminal deletions.

To help accomplish this goal, we have tested several lysis reagents with a library of protein constructs and have compared the solution chemical lysis methods to sonication. Here, we compare the solubility data obtained from a library of different size constructs of the ppsC's gene, originating from ACP domain and spanning up to the two adjacent domains; KR and ER. The lysis methods used include lysozyme, freeze-thaw cycles, Bugbuster, SoluLyse, and sonication. The goal of this experiment was to identify a chemical lysis method for our automated, HTP solubility assays that gives results that best match manual low throughput sonication.

\section{Materials and methods}

\section{Robotics integration}

Our integrated, high-throughput robotic system has previously been described [1]. Briefly, it includes a Biomek FX liquid handling robot, an ORCA ${ }^{\circledR}$ arm, a DTX plate reader equipped with filters allowing measurement of both absorbance and fluorescence (Beckman-Coulter, Fullerton, CA), a Cytomat 24 Hotel, Cytomat 2C incubators (ThermoFisher Scientific, Waltham, MA) and a Rotanta 46 ESC centrifuge (Hettich AG, Tuttlingen, Germany). For fluorescence imaging we used either an Illumatool lighting system LT-9500 (Lightools Research, Encinitas, CA) or Run Time Data Viewer, simulation software, version 3.0.0.9 (Beckman-Coulter, Fullerton, CA). Cultures were grown overnight in Innova 4230 refrigerated incubator shakers (New Brunswick Scientific, Edison, NJ). Sonication of the 96-well plates was performed manually using a Sonicator-ultrasonic processor XL20- 
20 (Misonix Inc., Farmingdale, NY). Biomek FX methods are designed using Biomek Software, version 3.2. Integration of all robotics components was controlled by SAMI Method Editor, version 3.5 (Beckman Coulter, Fullerton, CA).

\section{Expression library generation}

For the test of the different lysis methods using a liquid handling platform we used a library of 96 protein constructs representing the Acyl carrier protein (ACP) domain of the Mycobacterium tuberculosis Polyketide Synthase (ppsC) (Genbank accession number: CAB06099.1). The samples were prepared as previously described [12]. Following the library preparation, all plasmids were expressed in Escherichia coli BL21 (DE3) strain (Stratagene, La Jolla, CA). Overnight culture growth from the library's glycerol stock was performed in $175 \mu \mathrm{l}$ Luria-Bertani (LB) media supplemented with $7.5 \%$ glycerol and selective antibiotics spectinomycin $(75 \mu \mathrm{g} / \mathrm{ml})$ and kanamycin $(35 \mu \mathrm{g} / \mathrm{ml})$, standing at $32^{\circ} \mathrm{C}$ for $16 \mathrm{~h}$. Ten microlitres was used to inoculate $1 \mathrm{ml}$ of LB media supplemented with antibiotics in a 96 deep-well plate. Following $2 \mathrm{~h}$ outgrowth at $32^{\circ} \mathrm{C}, 350 \mathrm{rpm}$ in Innova 4230 refrigerated incubator shaker, protein expression was induced by anhydrotetracycline. Cultures were grown for additional $2 \mathrm{~h}$ at $32^{\circ} \mathrm{C}$, and then quenched using chloramphenicol (Sigma-Aldrich, St. Louis, MO). Equal volumes of expressed bacterial cultures $(175 \mu \mathrm{l})$ were transferred to $4 \times 96$-well microtitre plates and centrifuged at 4,000 rpm for $15 \mathrm{~min}$. The supernatant was removed from all plates. Bacterial pellets were dried and stored at $-80^{\circ} \mathrm{C}$ before lysis. Buffers used throughout the experimental procedure included either TNG buffer (100 mM Tris- $\mathrm{HCl}(\mathrm{pH} 7.4)$, $150 \mathrm{mM} \mathrm{NaCl}, 10 \%$ glycerol) or TN buffer (100 mM Tris$\mathrm{HCl}(\mathrm{pH} 7.4), 150 \mathrm{mM} \mathrm{NaCl}$ ). GFP 1-10 detector fragment reagent was prepared as described previously [12].

\section{Lysis methods}

Chemical cell lysis was performed using $120 \mu \mathrm{l}$ lysis solution containing either lysozyme (Sigma-Aldrich, St. Louis, MO) and 2 freeze-thaw cycles at $-80^{\circ} \mathrm{C}$, SoluLyse ${ }^{\circledR}$ in Tris buffer (Genlantis, San Diego, CA) or Bugbuster ${ }^{\circledR}$ protein extraction reagent (Novagen, EMD Chemicals Inc., San Diego, CA). For all commercial lysis reagents, and the lysozyme, manufacturer's protocols were followed with the addition of the Benzonase ${ }^{\circledR}$ (SigmaAldrich, St. Louis, MO). For manual sonication, $120 \mu \mathrm{l}$ of TN buffer was added to of 96-well plates containing centrifuged cells that were then sonicated using ultrasonic processor XL20-20, $3 \times 90 \mathrm{~s}, 50 \%$ cycle.
GFP 1-10 complementation and fluorescence data measurement

Following lysis, soluble and insoluble fractions were separated by centrifugation in Rotanta 46 RSC integrated centrifuge at $4,000 \mathrm{rpm}$ for $20 \mathrm{~min}, 4^{\circ} \mathrm{C}$. GFP $1-10 \mathrm{com}-$ plementation was achieved by the addition of $190 \mu \mathrm{l}$ of GFP 1-10 reagent into $40 \mu \mathrm{l}$ of the soluble fraction and $190 \mu \mathrm{l}$ of GFP $1-10$ reagent into $10 \mu \mathrm{l}$ of the solubilized pellet fraction (previously denatured using $60 \mu 19 \mathrm{M}$ urea in TNG). The split GFP can be used to measure as little as 0.2 pmol of protein in as little as 30 min using kinetics [14]. However, we chose to take advantage of the stability of the reconstituted GFP [12]. The final fluorescence value was measured after $24 \mathrm{~h}$. This eliminated possible time dependence of the readings and simplified calibration and measurement of many samples. GFP 1-10 complemented plates were incubated overnight at $4^{\circ} \mathrm{C}$ and the final fluorescence was measured using DTX reader. For fluorescence images, plates were illuminated using an Illumatool lighting system LT-9500. For the quantification of the soluble protein fraction, a set of eight different concentrations of the soluble GFP S11 tagged control protein, sulfite reductase, was obtained by serial dilution and used to generate a calibration curve as previously described [1]. The estimated expression yield (estimated $\mathrm{mg} / \mathrm{l}$ ) from the test protein cultures were each calculated using a calibration curve and the final fluorescence and calculated molecular weights of the protein fragments (all fragments were sequenced) as previously described [14].

Finally, the left and right ends of each construct and the measured soluble protein, insoluble protein, and fraction soluble were each mapped onto the genomic DNA sequence of the complete ppsC gene to visualize compact soluble domain boundaries.

\section{Results}

In this comparative study, we tested three different chemical lysis methods that can easily be automated and integrated into any HTP liquid handling robotic platform. Sonication was used as the standard of the comparison. A single 96 well plate containing picks from the ACP library was grown and used to inoculate four replicates for induction. All four methods were tested on expressed bacterial cell pellets originating from these replicates, under identical growth and expression conditions. For each plate, we used our split GFP to assay the soluble and insoluble protein fractions after disruption and centrifugation. Complementation with exogenous GFP 1-10 fragment resulted in a range of fluorescence depending on the solubility level of the tested protein constructs. Figure 1 

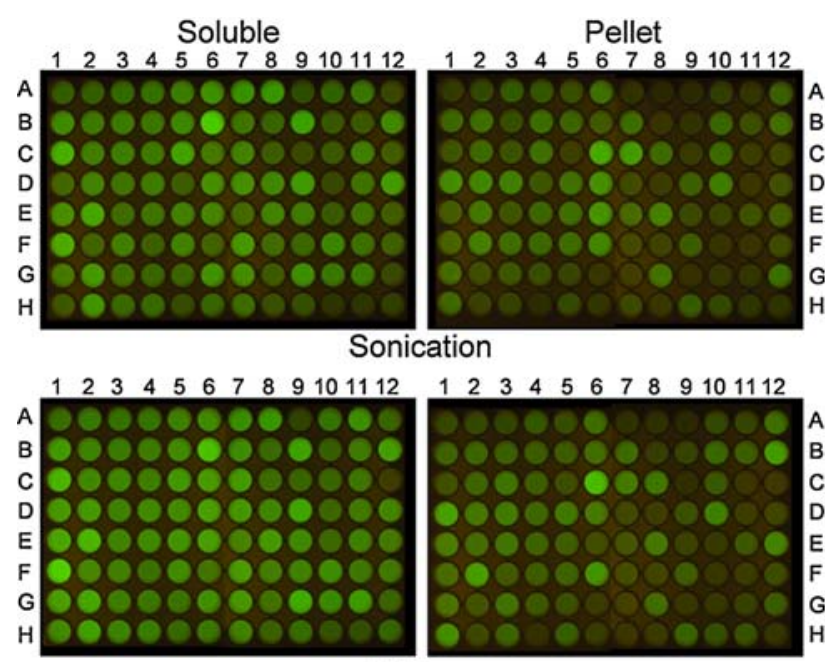

SoluLyse
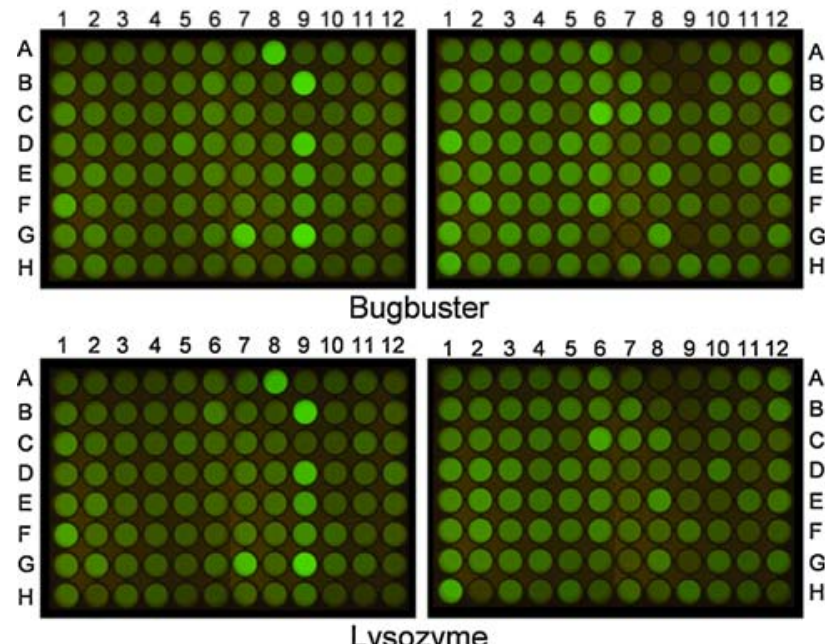

Fig. 1 Fluorescence data for 96 ACP domain ppsC library constructs obtained from four different lysis methods. Both soluble and pellet fractions are shown. Each well on four plates represents a single protein construct that was expressed using same conditions but lysed with one of the four lysis methods

represents the fluorescence images of both soluble and pellet fractions for all four lysis methods used in this study after their complementation with GFP 1-10. Each well on a given plate represents a single, unique ACP domain construct. The fluorescence signal for the same well position on different plates is indicative of the variation between the different lysis methods for the same construct. The sum of the fluorescence value for each soluble fraction and its corresponding pellet fraction represents the constructs total fluorescence. Because the absorbance value for each well is the same between four plates (data not shown), the difference between fluorescence values, and therefore relative solubilities, is reflective of the lysis method. Figure 2 shows the correlation between different chemical lysis methods and with sonication. The fluorescence data obtained for one lysis method was plotted against the data for the second lysis method. Figure 2a shows graphs and the correlation between sonication and different chemical lysis methods. The highest correlation value is for the SoluLyse reagent (correlation coefficient of 0.74). Both lysozyme and Bugbuster methods poorly correlate with sonication (correlation coefficients of 0.33 and 0.30 , respectively). Figure $2 \mathrm{~b}$ shows correlation between three chemical lysis methods. Lysozyme and Bugbuster methods have the highest correlation with each other (correlation coefficient of 0.97). Table 1 combines the total expression and the percentage of the released soluble protein using four lysis methods. The position on the plate, length of the construct, molecular weight, total expression $(\mathrm{mg} / \mathrm{l})$, and the percentage of the soluble fraction is presented. Figure 3 maps the ACP domain constructs onto the ppsC sequence used in this study. The six colors represent fragments with increasing solubility percentage $(0-17 \%$ red, $17-34 \%$ orange, $34-50 \%$ yellow, $50-67 \%$ green, $67-84 \%$ dark blue and $84-100 \%$ light blue (expressed as the percentage of the total protein expression). The pattern of solubility in panels A and B of Fig. 3 are quite similar, as are those in panels $\mathrm{C}$ and $\mathrm{D}$. This is consistent with the results shown in Fig. 2, where the sonication and SoluLyse ${ }^{\circledR}$ methods are correlated. Neither the BugBuster ${ }^{\circledR}$ nor lysozyme methods correlate with sonication, suggesting a protein dependent bias of solubility or lysis efficiency relative to sonication. The strong correlation of BugBuster ${ }^{\circledR}$ with lysis by freeze thaw suggests a similar mode of bias. Table 1 summarizes the calculations of the total expression yield and the success of the lysis method as a function of percentage of the soluble fraction released from the bacterial cells. Despite the fact that all plates contained the same amount of protein expressed before the lysis, there is a variation in total expression yield between the methods as detected by the complementation with GFP 1-10. These results suggest that chemical reagents not only rupture the bacterial cells but also have an effect on the proteins' physicochemical properties by affecting the exposure of the GFP S11 tag on the construct to complementation by GFP 1-10 and thus final fluorescence. The results clearly indicate that none of the chemical methods tested are identical to sonication. SoluLyse ${ }^{\circledR}$ reagent was found to be the most similar to sonication. Lysozyme and Bugbuster ${ }^{\circledR}$ lysis showed poor correlation with sonication under the conditions tested. However, these methods produce results similar to each other, especially for smaller protein constructs. The noticeable outliers are found in Fig. 1, positions A8, B9, D9, G7 and G9. The same constructs can be seen in Fig. 3, as the only fragments 50 percent or more soluble (dark blue and light blue colors). These are the 
Table 1 Comparison of expression yield and lysis method effectiveness using four lysis methods

\begin{tabular}{|c|c|c|c|c|c|c|c|c|c|c|}
\hline \multirow[t]{2}{*}{$\mathrm{R}: \mathrm{C}$} & \multirow[t]{2}{*}{ aa } & \multirow[t]{2}{*}{ MW } & \multicolumn{2}{|l|}{ Sonication } & \multicolumn{2}{|l|}{ Solulyse } & \multicolumn{2}{|l|}{ Bugbuster } & \multicolumn{2}{|l|}{ Lysozyme } \\
\hline & & & Total (mg/l) & Sol (\%) & Total (mg/l) & Sol (\%) & Total (mg/l) & Sol (\%) & Total (mg/l) & Sol (\%) \\
\hline A1 & 436 & 536,81 & 34 & 51 & 50 & 52 & 60 & 27 & 53 & 29 \\
\hline B1 & 442 & 544,40 & 61 & 47 & 68 & 56 & 75 & 28 & 61 & 29 \\
\hline $\mathrm{C} 1$ & 411 & 506,04 & 55 & 70 & 59 & 76 & 59 & 37 & 53 & 39 \\
\hline D1 & 471 & 583,40 & 67 & 20 & 101 & 31 & 119 & 18 & 64 & 24 \\
\hline E1 & 420 & 516,74 & 36 & 57 & 63 & 50 & 64 & 30 & 52 & 31 \\
\hline $\mathrm{F} 1$ & 412 & 507,21 & 47 & 63 & 70 & 69 & 81 & 37 & 62 & 43 \\
\hline G1 & 439 & 540,45 & 42 & 38 & 61 & 51 & 89 & 22 & 57 & 28 \\
\hline H1 & 459 & 568,04 & 42 & 25 & 89 & 32 & 113 & 18 & 94 & 15 \\
\hline $\mathrm{A} 2$ & 442 & 544,40 & 49 & 43 & 65 & 56 & 86 & 24 & 56 & 30 \\
\hline B2 & 438 & 539,13 & 69 & 37 & 69 & 46 & 92 & 23 & 60 & 24 \\
\hline $\mathrm{C} 2$ & 425 & 523,83 & 55 & 42 & 58 & 49 & 83 & 24 & 55 & 27 \\
\hline D2 & 439 & 540,45 & 74 & 32 & 77 & 47 & 89 & 24 & 71 & 21 \\
\hline E2 & 423 & 520,43 & 71 & 50 & 76 & 62 & 82 & 28 & 69 & 26 \\
\hline G2 & 412 & 507,21 & 45 & 68 & 56 & 71 & 61 & 36 & 56 & 36 \\
\hline $\mathrm{H} 2$ & 403 & 496,44 & 47 & 71 & 58 & 74 & 76 & 31 & 17 & 44 \\
\hline A3 & 435 & 535,62 & 73 & 40 & 66 & 54 & 95 & 22 & 56 & 27 \\
\hline B3 & 435 & 535,62 & 58 & 54 & 73 & 45 & 71 & 30 & 62 & 24 \\
\hline $\mathrm{C} 3$ & 442 & 544,40 & 53 & 53 & 86 & 40 & 84 & 25 & 60 & 26 \\
\hline D3 & 425 & 523,83 & 77 & 27 & 82 & 36 & 90 & 21 & 66 & 20 \\
\hline E3 & 426 & 524,72 & 41 & 45 & 73 & 40 & 86 & 25 & 59 & 23 \\
\hline F3 & 434 & 534,73 & 64 & 40 & 53 & 51 & 84 & 23 & 53 & 29 \\
\hline G3 & 426 & 524,72 & 46 & 43 & 74 & 38 & 93 & 20 & 52 & 25 \\
\hline H3 & 433 & 533,08 & 43 & 52 & 81 & 47 & 94 & 22 & 54 & 27 \\
\hline $\mathrm{A} 4$ & 436 & 536,81 & 68 & 46 & 73 & 46 & 88 & 24 & 56 & 27 \\
\hline B4 & 439 & 540,45 & 75 & 37 & 68 & 47 & 86 & 24 & 63 & 24 \\
\hline D4 & 443 & 545,86 & 53 & 50 & 73 & 48 & 81 & 28 & 53 & 27 \\
\hline E4 & 435 & 535,62 & 61 & 39 & 71 & 50 & 95 & 22 & 60 & 24 \\
\hline G4 & 439 & 540,45 & 50 & 44 & 65 & 48 & 92 & 21 & 62 & 24 \\
\hline $\mathrm{H} 4$ & 412 & 507,21 & 38 & 70 & 54 & 75 & 61 & 33 & 46 & 38 \\
\hline A5 & 419 & 515,69 & 54 & 56 & 58 & 55 & 70 & 27 & 50 & 31 \\
\hline B5 & 434 & 534,73 & 59 & 49 & 59 & 52 & 80 & 24 & 63 & 24 \\
\hline C5 & 411 & 506,04 & 47 & 79 & 49 & 68 & 46 & 43 & 44 & 40 \\
\hline D5 & 436 & 536,93 & 49 & 24 & 74 & 40 & 103 & 29 & 60 & 22 \\
\hline E5 & 442 & 544,40 & 63 & 41 & 63 & 56 & 84 & 24 & 64 & 24 \\
\hline F5 & 435 & 535,62 & 67 & 42 & 69 & 51 & 87 & 24 & 54 & 25 \\
\hline A6 & 385 & 473,35 & 62 & 47 & 63 & 43 & 87 & 21 & 54 & 23 \\
\hline B6 & 412 & 507,21 & 69 & 75 & 78 & 64 & 71 & 31 & 59 & 34 \\
\hline C6 & 459 & 568,04 & 91 & 20 & 128 & 24 & 137 & 14 & 92 & 15 \\
\hline D6 & 439 & 540,45 & 74 & 33 & 67 & 47 & 74 & 26 & 53 & 26 \\
\hline E6 & 439 & 540,45 & 77 & 28 & 47 & 71 & 83 & 24 & 59 & 24 \\
\hline F6 & 567 & 701,78 & 81 & 20 & 104 & 24 & 133 & 16 & 81 & 18 \\
\hline G6 & 411 & 506,04 & 42 & 78 & 44 & 76 & 53 & 43 & 47 & 36 \\
\hline H6 & 412 & 507,21 & 35 & 59 & 48 & 65 & 61 & 29 & 42 & 32 \\
\hline A7 & 411 & 506,04 & 52 & 77 & 54 & 77 & 53 & 42 & 42 & 44 \\
\hline B7 & 439 & 540,45 & 55 & 36 & 67 & 46 & 88 & 24 & 59 & 25 \\
\hline $\mathrm{C} 7$ & 425 & 523,83 & 81 & 26 & 69 & 44 & 86 & 21 & 54 & 25 \\
\hline D7 & 404 & 497,45 & 32 & 68 & 36 & 72 & 41 & 42 & 33 & 43 \\
\hline
\end{tabular}


Table 1 continued

\begin{tabular}{|c|c|c|c|c|c|c|c|c|c|c|}
\hline \multirow[t]{2}{*}{$\mathrm{R}: \mathrm{C}$} & \multirow[t]{2}{*}{ aa } & \multirow[t]{2}{*}{ MW } & \multicolumn{2}{|l|}{ Sonication } & \multicolumn{2}{|l|}{ Solulyse } & \multicolumn{2}{|l|}{ Bugbuster } & \multicolumn{2}{|l|}{ Lysozyme } \\
\hline & & & Total (mg/l) & Sol (\%) & Total (mg/l) & Sol (\%) & Total (mg/l) & Sol (\%) & Total (mg/l) & Sol $(\%)$ \\
\hline E7 & 365 & 451,10 & 29 & 32 & 27 & 58 & 38 & 33 & 27 & 37 \\
\hline F7 & 395 & 487,33 & 29 & 74 & 33 & 72 & 40 & 38 & 32 & 39 \\
\hline G7 & 160 & 198,36 & 11 & 78 & 13 & 75 & 21 & 89 & 18 & 76 \\
\hline A8 & 152 & 189,75 & 20 & 87 & 25 & 79 & 38 & 94 & 33 & 87 \\
\hline B8 & 401 & 493,61 & 24 & 64 & 32 & 58 & 35 & 38 & 29 & 37 \\
\hline D8 & 404 & 497,45 & 31 & 74 & 33 & 72 & 38 & 42 & 33 & 40 \\
\hline E8 & 436 & 536,81 & 64 & 34 & 71 & 46 & 87 & 23 & 63 & 21 \\
\hline F8 & 358 & 443,45 & 19 & 60 & 26 & 66 & 35 & 38 & 26 & 36 \\
\hline G8 & 486 & 600,14 & 49 & 24 & 57 & 35 & 97 & 19 & 50 & 25 \\
\hline H8 & 298 & 367,89 & 14 & 52 & 19 & 57 & 27 & 32 & 20 & 41 \\
\hline A9 & 288 & 358,26 & 19 & 60 & 18 & 57 & 29 & 45 & 23 & 42 \\
\hline B9 & 152 & 189,75 & 22 & 82 & 28 & 73 & 42 & 95 & 38 & 88 \\
\hline C9 & 454 & 559,97 & 24 & 43 & 26 & 74 & 43 & 32 & 28 & 38 \\
\hline D9 & 152 & 189,75 & 25 & 57 & 25 & 69 & 39 & 70 & 28 & 72 \\
\hline G9 & 152 & 189,75 & 15 & 84 & 23 & 84 & 37 & 94 & 31 & 87 \\
\hline A10 & 443 & 545,86 & 46 & 51 & 65 & 52 & 68 & 35 & 48 & 38 \\
\hline B10 & 465 & 575,63 & 58 & 27 & 67 & 29 & 82 & 27 & 56 & 25 \\
\hline $\mathrm{C} 10$ & 488 & 602,36 & 60 & 25 & 52 & 40 & 78 & 21 & 46 & 27 \\
\hline D10 & 395 & 486,44 & 56 & 14 & 70 & 24 & 85 & 17 & 55 & 20 \\
\hline E10 & 368 & 454,77 & 25 & 47 & 26 & 63 & 33 & 39 & 25 & 38 \\
\hline F10 & 411 & 506,04 & 40 & 76 & 48 & 78 & 52 & 46 & 43 & 39 \\
\hline G10 & 412 & 507,21 & 40 & 70 & 51 & 75 & 54 & 38 & 52 & 33 \\
\hline H10 & 465 & 575,63 & 40 & 21 & 59 & 35 & 86 & 19 & 50 & 22 \\
\hline A11 & 411 & 506,04 & 36 & 69 & 58 & 68 & 46 & 41 & 49 & 31 \\
\hline C11 & 406 & 499,75 & 30 & 69 & 26 & 73 & 38 & 45 & 43 & 34 \\
\hline D11 & 112 & 141,03 & 7 & 70 & 8 & 69 & 9 & 45 & 8 & 40 \\
\hline E11 & 406 & 499,71 & 36 & 48 & 45 & 53 & 58 & 38 & 44 & 25 \\
\hline F11 & 395 & 487,33 & 26 & 71 & 33 & 68 & 36 & 40 & 29 & 39 \\
\hline G11 & 410 & 504,73 & 37 & 71 & 62 & 74 & 51 & 42 & 48 & 35 \\
\hline H11 & 487 & 601,31 & 29 & 33 & 60 & 32 & 84 & 28 & 49 & 21 \\
\hline A12 & 486 & 600,14 & 39 & 23 & 60 & 30 & 65 & 25 & 54 & 21 \\
\hline B12 & 412 & 507,21 & 47 & 44 & 86 & 36 & 71 & 22 & 50 & 20 \\
\hline C12 & 452 & 557,19 & 19 & 50 & 9 & 42 & 37 & 35 & 31 & 36 \\
\hline D12 & 410 & 504,73 & 38 & 68 & 28 & 70 & 50 & 38 & 44 & 34 \\
\hline F12 & 452 & 557,19 & 27 & 46 & 36 & 58 & 42 & 32 & 35 & 34 \\
\hline G12 & 487 & 601,03 & 53 & 19 & 49 & 38 & 75 & 22 & 53 & 22 \\
\hline
\end{tabular}

Expression yield is represented as $\mathrm{mg} / \mathrm{ml}$ of total protein while lysis method effectiveness is expressed as the percentage of released soluble protein (\% sol). The position on the plate (R:C), the number of amino acids (aa), and the construct molecular weight (MW) is presented for all constructs

smallest fragments covering primarily the ACP domain (fragments size range 152-160 amino acids). It is likely that these smaller proteins are relatively insensitive to the choice of lysis method. Interestingly, except for these small fragments, all of the remaining protein constructs show poor solubility $(<50 \%)$ relative to sonication or SoluLyse ${ }^{\circledR}$.

\section{Discussion and conclusions}

Structural genomics laboratories around the world are striving to develop robust expression screening methods on a small scale using liquid handling platforms that would identify highly soluble proteins amenable for scale-up production and structural analysis. In some cases, the 

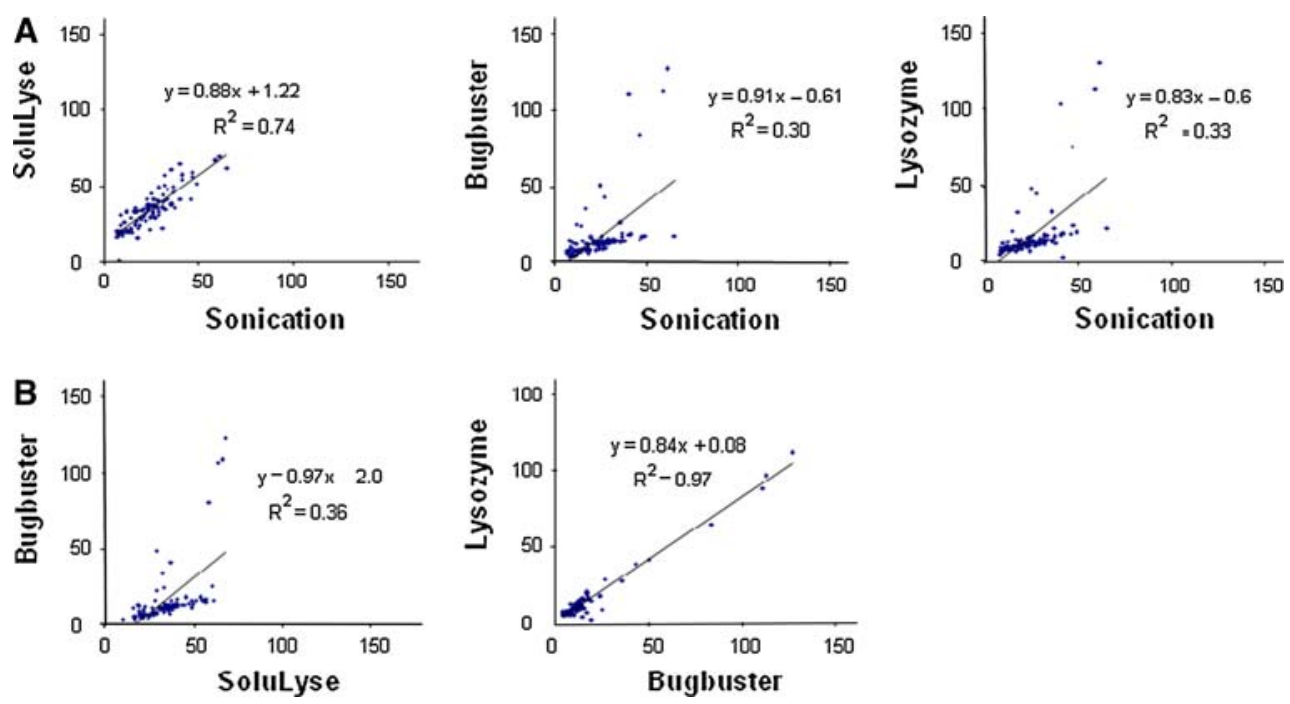

Fig. 2 a Correlation analysis between three different chemical lysis methods and sonication using the fluorescence values (all $F$-values shown are E + 06). Lysozyme, SoluLyse and Bugbuster reagents were used to chemically disrupt bacterial cells for the release of the expressed proteins. Similarly, the same constructs were lysed mechanically by sonication ("gold" standard). b Additionally, correlation between three chemical methods is also shown. From the plotted data SoluLyse reagent shows to be most similar to sonication. Correlation value ( $R$ value $)$ for lysozyme and the Bugbuster shows that these two methods are also most similar

measure as insoluble, compressing most of the data points into a narrow region (Fig. 2). Consequently, most of the fitting power in the correlation plot between the Bugbuster ${ }^{\circledR}$ and lysozyme is largely based on a small subset of the proteins being effectively released (the smaller fragments). In contrast, most of the proteins are successfully released by sonication, without bias to size of the protein. It is also important to note that the solubility of the fragments spanning the three ppsC domains is also related to the known boundaries of the domains. Figure 3 shows that most of the fragments reach their highest solubility values when approaching the boundary of the linker region between the ER and KR domains. Larger fragments that originate in the ER domain are predominantly $<50 \%$ soluble. The solubility of these fragments can be affected by the larger size of the fragment itself and/or the ER domain's incomplete or disrupted folding affecting the folding of the rest of the protein construct. Because SoluLyse ${ }^{\circledR}$ is well correlated with sonication indicates that this chemical method is acceptable for this work (Fig. 2). This can also be visualized in Fig. 3, where the most soluble Solulyse and sonication fragments indicate the boundary between the ER and KR domains.

In conclusion, the purpose of this study is to evaluate several chemical lysis methods for the release of the soluble proteins from their bacterial expression host. When bacterial cells preparations are lysed on a small scale, majority of laboratories use the sonication as the most efficient method for complete cell rupture. Moving from laboratory bench to the HTP robotics platform often creates several bottlenecks, one of them being the 


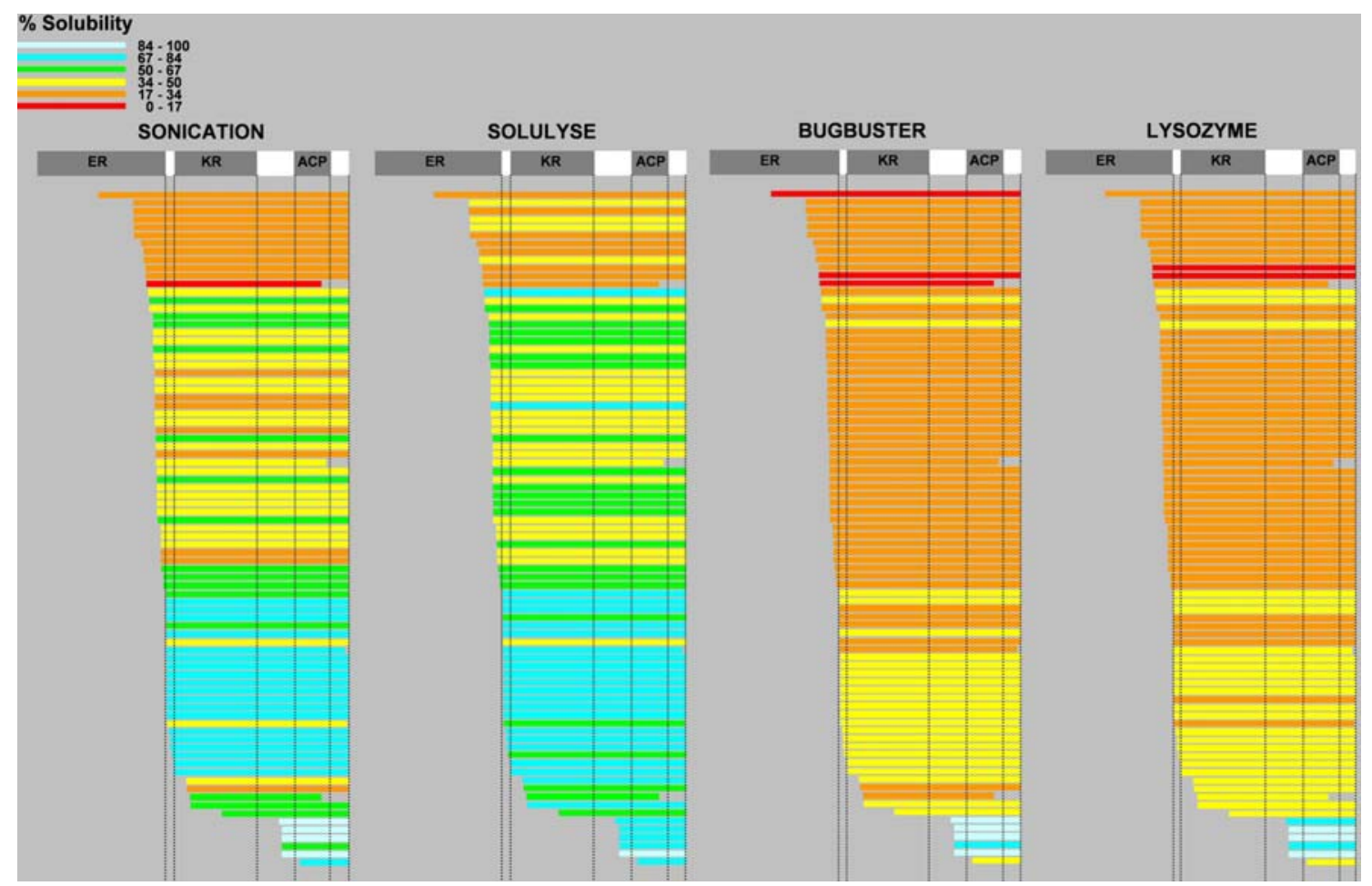

Fig. 3 Mapping of the solubility data obtained using four different lysis methods. Each construct is sequenced, assigned solubility level and mapped onto the region of the ppsC gene containing ACP domain. Colors of the bars represent percentage solubility as calculated by comparison of soluble and pellet fractions. Colors red, orange and yellow represent constructs with $<50 \%$ calculated soluble fraction. Colors green, dark and light blue represent constructs with above 50\% solubility. Vertical dotted lines represent the known boundaries of the $\mathrm{ACP}, \mathrm{KR}$ and ER domains. To simplify the view of the map, only three-C-terminal domains of the ppsC gene are shown availability of the high number of samples processing sonicators and/or their integration with the existing robotics platforms. For this reason, most HTP laboratories develop an in-house chemical lysis method, use commercially available chemical reagents with or without any modification, or include a manual sonication step, where the plates are moved away from the robotics platform and processed using stand alone sonicators. In our comparison study, SoluLyse ${ }^{\circledR}$ has shown to be the method with the highest correlation to sonication. Interestingly, widely used lysozyme and Bugbuster ${ }^{\circledR}$ failed in most our cases to completely release the soluble protein. In applications such as protein domain trapping, the precise solubility information is critical in predicting the boundaries of the domains. It is therefore of outmost importance that the lysis method used in such applications be non-perturbing. When sonication is inconvenient, or difficult to apply to many samples, we find SoluLyse ${ }^{\circledR}$ to be an acceptable alternative for the ACP fragment proteins as well as many other constructs we have examined to date.

Acknowledgments This work was supported by the NIH Protein Structure Initiative (U54 GM074946-01US, “Integrated Center for Structure and Function Innovation").
Open Access This article is distributed under the terms of the Creative Commons Attribution Noncommercial License which permits any noncommercial use, distribution, and reproduction in any medium, provided the original author(s) and source are credited.

\section{References}

1. Listwan P, Terwilliger TC, Waldo GS (2009) Automated, highthroughput platform for protein solubility screening using a splitGFP system. J Struct Funct Genomics 10:47-55

2. Berrow NS, Büssow K, Coutard B, Diprose J, Ekberg M, Folkers GE, Levy N, Lieu V, Owens RJ, Peleg Y, Pinaglia C, QuevillonCheruel S, Salim L, Scheich C, Vincentell R, Busso D (2006) Recombinant protein expression and solubility screening in Escherichia coli: a comparative study. Acta Crystallogr D Biol Crystallogr 62:1218-1226

3. Kim Y, Dementieva I, Zhou M, Wu R, Lezondra L, Quartey P, Joachimiak G, Korolev O, Li H, Joachimiak A (2004) Automation of protein purification for structural genomics. J Struct Funct Genomics 5:111-118

4. Mayer MR, Dailey TA, Baucom CM, Supernak JL, Grady MC, Hawk HE, Dailey HA (2004) Expression of human proteins at the southeast collaboratory for structural genomics. J Struct Funct Genomics 5:159-165

5. Vincentelli R, Canaan S, Offant J, Cambillau C, Bignon C (2005) Automated expression and solubility screening of His-tagged proteins in 96-well format. Anal Biochem 346:77-84 
6. Nguyen H, Martinez B, Oganesyan N, Kim R (2004) An automated small-scale protein expression and purification screening provides beneficial information for protein production. J Struct Funct Genomics 5:23-27

7. Finley JB, Qiu SH, Luan CH, Luo M (2003) Structural genomics for caenorhabditis elegans: high throughput protein expression analysis. Protein Expr Purif 34:49-55

8. Tegel H, Steen J, Konrad A, Nikdin H, Pettersson K, Stenvall M, Tourle S, Wrethagen U, Xu LL, Yderland L, Uhlén M, Hober S, Ottosson J (2009) High-throughput protein production-lessons from scaling up from 10 to 288 recombinant proteins per week. Biotechnol J 4:51-57

9. Mitraki A, King J (1989) Protein folding intermediates and inclusion body formation. Nat Biotechnol 7:690-697

10. Feliu JH, Cubarsi R, Villaverde A (1998) Optimized release of recombinant proteins by ultracentrifugation of $E$. coli cells. Biotech Bioeng 58(5):536-540

11. Ho CW, Chew TK, Ling TC, Kamaruddin S, Tan WS, Tey BT (2006) Efficient mechanical cell disruption of Escherichia coli by an ultrasonicator and recovery of intracellular hepatitis B core antigen. Process Biochem 41:1829-1834

12. Cabantous S, Waldo GS (2006) In vivo and in vitro protein solubility assays using split GFP. Nat Methods 3:845-854

13. Cabantous S, Terwilliger TC, Waldo GS (2005) Protein tagging and detection with engineered self-assembling fragments of green fluorescent protein. Nat Biotechnol 23:102-107

14. Cabantous S, Pédelacq JD, Mark B, Naranjo C, Terwilliger TC, Waldo GC (2005) Recent advances in GFP folding reporter and split-GFP solubility reporter technologies. Application to improving the folding and solubility of recalcitrant proteins from Mycobacterium tuberculosis. J Struct Funct Genomics 6:113-118

15. Waldo GS, Standish BM, Berendzen J, Terwilliger TC (1999) Rapid protein folding assay using green fluorescent protein. Nat Biotechnol 17:691-695

16. Pédelacq JD, Cabantous S, Tran T, Terwilliger TC, Waldo GS (2006) Engineering and characterization of a superfolder green fluorescent protein. Nat Biotechnol 24:79-88 